\title{
Objective measurement of contrast sensitivity function using contrast sweep visual evoked responses
}

\author{
Jacqueline M Lopes de Faria, Osamu Katsumi, Mikki Arai, Tatsuo Hirose
}

\begin{abstract}
Aim/background-The contrast sensitivity function (CSF) measurement provides information that is not accessible by standard visual acuity determinations. The contrast sweep pattern reversal visual evoked responses (CSVER) technique was used to objectively measure the CSF in clinical practice.

Methods-The contrast thresholds were measured at five spatial frequencies in $\mathbf{1 0}$ normal subjects. The CSVER were recorded with sinusoidally modulated vertical gratings at 10 contrast levels $(96,64,48$, $32,16,8,4,2,1$, and $0.5 \%$ ) presented in five spatial frequencies $(0.5,1.0,2.0,4.0$, and 8.0 cycles per degree). Each of 10 contrast levels was displayed for 2 seconds at the desired spatial frequency. The CSVER amplitudes at the second harmonic were calculated by discrete Fourier transform. The results were compared with those obtained using a psychophysical method. Results-An inverted U-shaped CSF which peaked at 2.0 cycles per degree with a contrast sensitivity of 34.5 (contrast, $2.9 \%$ ) was observed. The CSF assessed electrophysiologically was 0.62 to $0.79 \mathrm{log}$ units lower than the sensitivity measured using the psychophysical method. However, the overall shapes were highly correlated.
\end{abstract}

Conclusion-One can objectively measure CSF with CSVER and this may be useful in patients in whom the psychophysical method is limited.

(Br f Ophthalmol 1998;82:168-173)

The contrast sensitivity function (CSF) is a basic measurement of human spatial vision that provides a clinical evaluation of visual function over a wide range of spatial frequencies. CSF measurement is important because it reflects the subject's visual ability in his or her low contrast living environment in which there are numerous objects. The CSF concept, developed by Campbell and Robson, ${ }^{1}$ plays an important role in psychophysical $^{1}$ and electrophysiological ${ }^{2}$ studies of the visual system. In addition, CSF measurements have contributed to the diagnosis and understanding of visual disorders in diseases such as multiple sclerosis, amblyopia, and diabetes mellitus. ${ }^{3-7}$

The CSF measurement provides information that is not accessible by standard visual acuity determinations typically obtained with high contrast optotypes. ${ }^{6-9}$ The clinical application of CSF in ophthalmology has been delayed because the complexity of the method limited it to use with alert and cooperative subjects. Recently, some studies have demonstrated much simpler and less time consuming psychophysical CSF methodologies. ${ }^{70}$ However, when assessing vision in a paediatric population or in non-verbal or mentally deficient patients in whom these tests may not be effective, an "objective" test for measuring CSF is desirable. The pattern reversal visual evoked response (PVER), ${ }^{11}$ which primarily reflects macular function, ${ }^{12-16}$ is useful for CSF measurement. ${ }^{17-20}$ Although the results of electrophysiological measurements of CSF roughly parallel the psychophysical determinations, the use of the standard PVER for CSF measurement is time consuming. In addition, the CSF derived from VER is less sensitive than the psychophysical test ${ }^{18}{ }^{21}$ and there may be irregularities in the VER signals, ${ }^{22}$ making the test impractical for clinical use.

The spatial frequency sweep PVER (SPVER), introduced by Regan ${ }^{23}$ and later by Tyler et $a l^{24}$ allows visual sensory threshold evaluation within a short time. Although the spatial frequency sweep PVER analyses the visual function in the spatial domain, this algorithm can be used to study the visual function at the contrast domain by sweeping the stimulus at different contrast levels. In the present study, instead of sweeping spatial frequencies, a large number of contrast stimuli were displayed consecutively without interruption for 20 seconds at each spatial frequency. We thus obtained the contrast threshold at each spatial frequency within a very short time. This methodology is referred to here as contrast sweep VER (CSVER).

In the present investigation, we measured the contrast thresholds of normal adults by CSVER, analysed the effect of contrast ratio on the amplitude response at different spatial frequencies, and compared the findings with the subjective (conventional) CSF.

\section{Materials and methods}

PATIENTS

Ten ophthalmologically normal volunteers (six men, four women; age range 25-48 years) participated in this study. All subjects had a corrected visual acuity of $20 / 20$ or better at the time of the recording. Each subject underwent monocular electrophysiological and psychophysical testing of a randomly selected eye with an undilated pupil in a dark room. Before the 

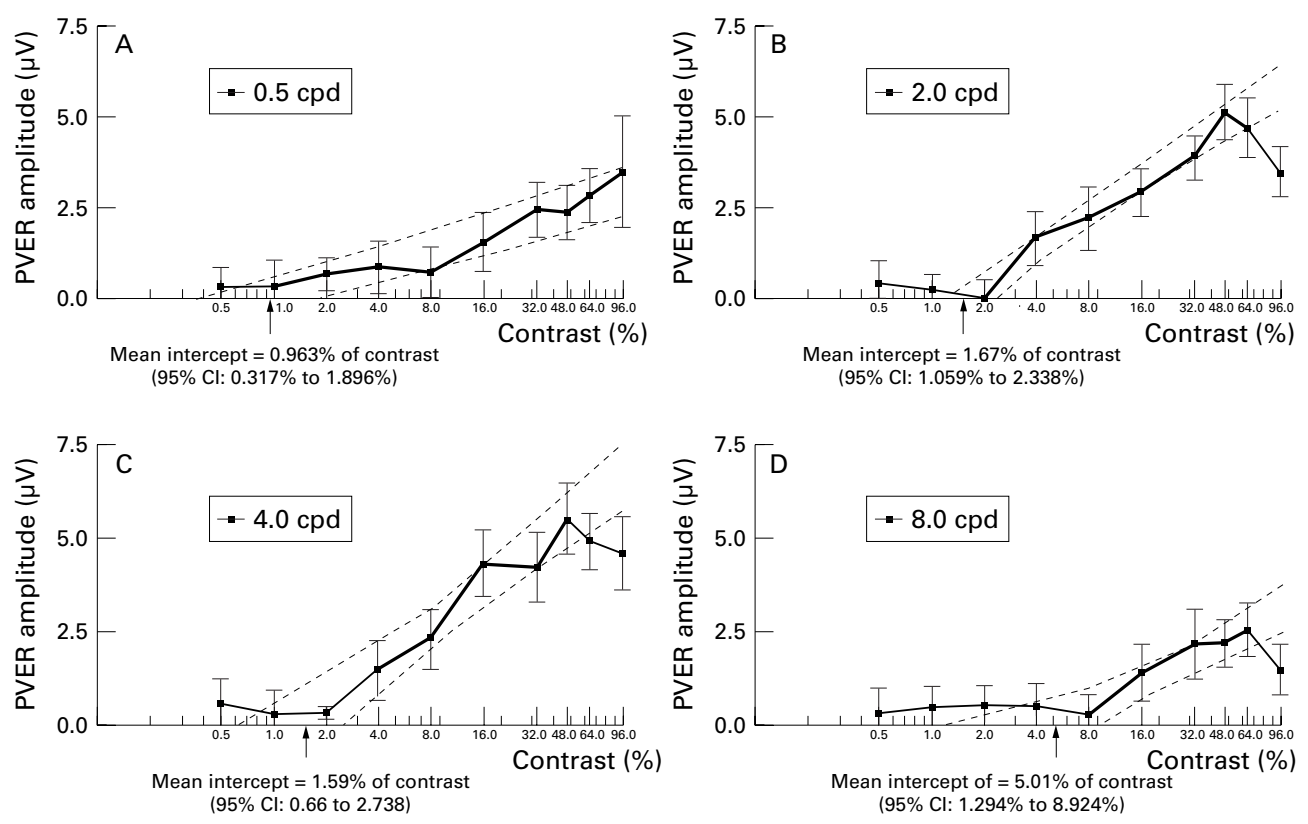

Figure 1 (A-D) Contrast sweep visual evoked response (CSVER) from a normal female subject in four spatial frequencies. In each figure, the mean VER amplitude-contrast function with the $95 \%$ confidence interval (CI) was plotted at each of the 10 different contrasts. The broken lines indicate the best fit linear regression with the 95\% CI in each spatial frequency. The contrast threshold was determined by extrapolating to $0 \mu V$ on the $x$ axis.

measurements were recorded, the procedures were fully explained to each subject, and informed consent was obtained in all cases. This study was carried out in conformity with the tenets of the Declaration of Helsinki.

RECORDING CSVER

The stimulus pattern was displayed on a 19 inch, high resolution television monitor (P7A24K-931, Pixelink) with a spatial resolution of 960 horizontal raster lines. The overall stimulus field was $25 \mathrm{~cm} \times 25 \mathrm{~cm}$. The stimulus field size subtended a visual angle of $14^{\circ} \times$ $14^{\circ}$ at the testing distance of $100 \mathrm{~cm}$. The mean luminance was maintained at $50 \mathrm{~cd} / \mathrm{m}^{2}$. The CSVER were recorded using standard electroencephalogram cup occipital electrodes positioned at $\mathrm{Oz} / \mathrm{Pz}$ (the electrodes were placed anterior to the inion, $\mathrm{Oz}$ at $10 \%$ of the

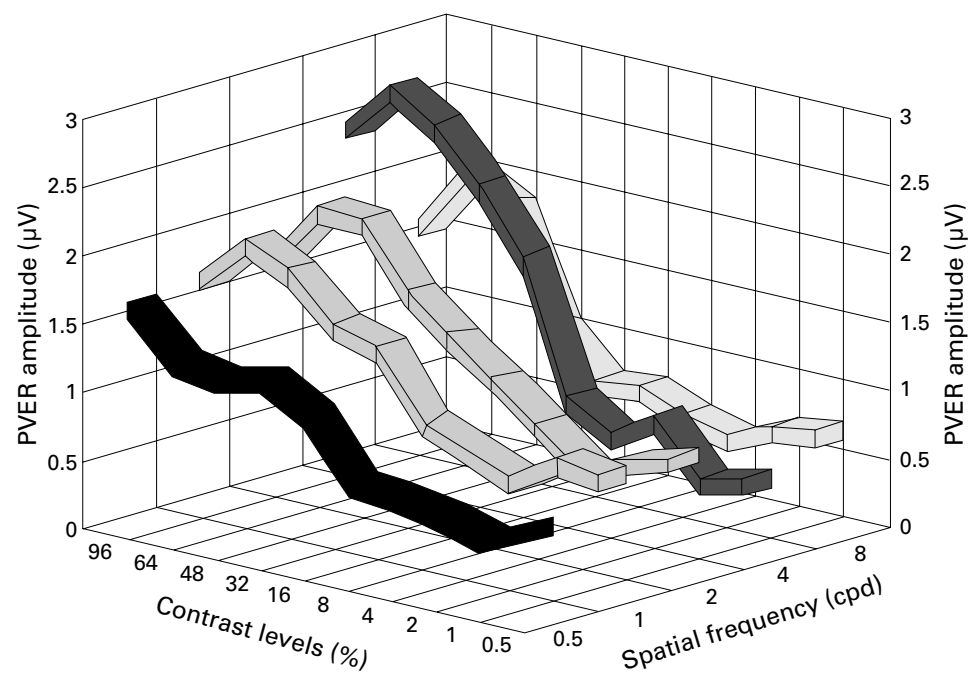

Figure 2 The mean CSVER amplitude-contrast level function at five spatial frequencies in 10 normal volunteers. $c$ pd $=$ cycles per degree; PVER = pattern visual evoked responses. inion-nasion measurement and $\mathrm{Pz}$ at $30 \%$ of inion-nasion measurement), amplified with a $0.5-100 \mathrm{~Hz}$ bandwidth isolated differential amplifier (Model Enfant 4010, Neuroscientific Corp, Farmingdale, NY, USA), digitised at $180 \mathrm{~Hz}$, and harmonically synchronised (phase coherent) to the stimulus presentation. The digitised samples were divided into analysis records (epochs) of 180 points each (that is, 1 second). Each record was analysed using discrete Fourier transform (DFT), the values of which were vector averaged and converted into polar form to yield the appropriate magnitude and phase for the second harmonic frequencies $(12 \mathrm{~Hz})$ of the mean value of the Fourier coefficients. Sinusoidally modulated vertical gratings of 10 different contrast levels ranging from the highest $(96 \%)$ to the lowest $(0.5 \%)$ level were swept at five spatial frequencies: $0.5,1.0,2.0,4.0$, and 8.0 cycles per degree (cpd). The contrast levels measured were $96,64,48,32,16,8,4,2,1$, and $0.5 \%$. The pattern reversal rate was fixed at 12 reversals per second $(6 \mathrm{~Hz})$. Each of 10 contrast levels was displayed for 2 seconds, for a total recording time of 20 seconds at each of the five spatial frequencies. The mean contrast threshold and the $95 \%$ confidence interval (CI) were calculated using the manufacturer's software. The results were immediately displayed on the video monitor of a personal computer.

\section{DETERMINING THE CONTRAST THRESHOLD} ELECTROPHYSIOLOGICALLY

The contrast threshold was determined as follows ${ }^{25}$ : after the recordings, two points were manually selected for analysis, one at the highest contrast level which records the first peak of PVER amplitude and the other at the lowest contrast level which produces the minimum 

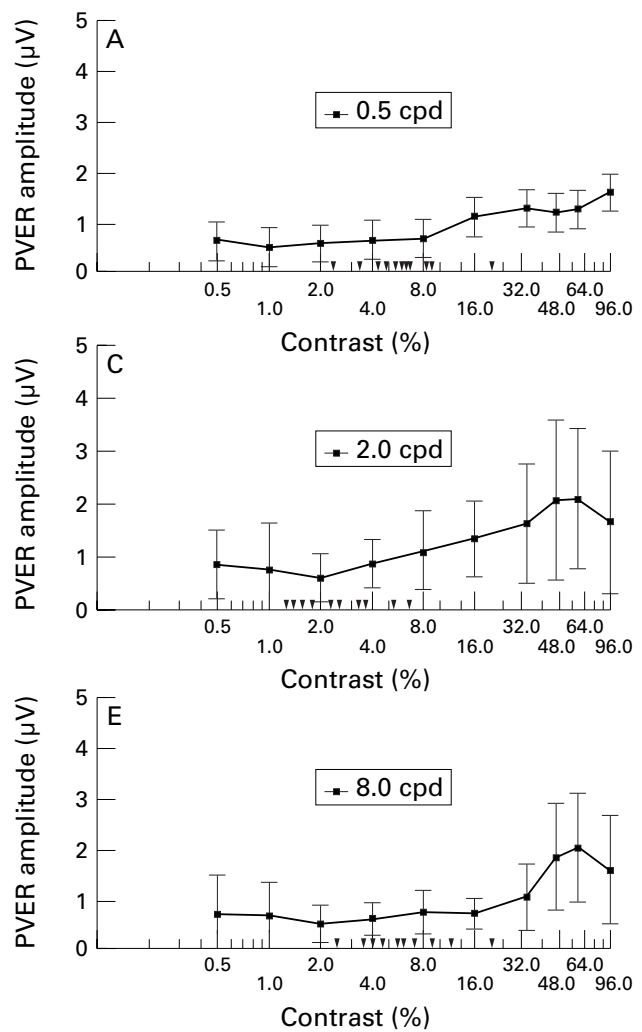

level separate from the noise on the descending curve of the CSVER amplitude contrast function. Derived from an extensive empirical data set, any evoked response is at least three times greater than the average noise level and the phase of response is steady (within 20 degrees) or slightly leading the stimulus (no more than 90 degrees of phase shift between points). ${ }^{26}$ The best fit regression line (within 95\% confidence interval, because the CSVER function is not always a simple linear function of log contrast) was then drawn by the Enfant system between these two points and included all datapoints between them. The contrast threshold was determined on the intersection of the linear regression line with $0 \mu \mathrm{V}$. The determi-

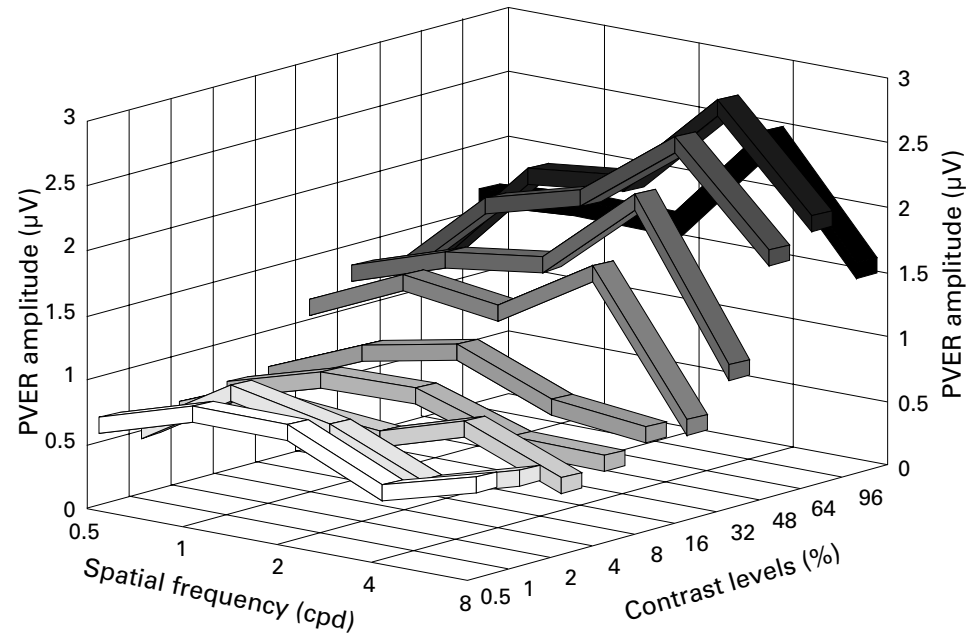

Figure 4 The CSVER amplitude-spatial frequency function at 10 contrast levels in 10 normal volunteers. $P V E R=$ pattern reversal visual evoked responses; $c p d=$ cycles per degree.
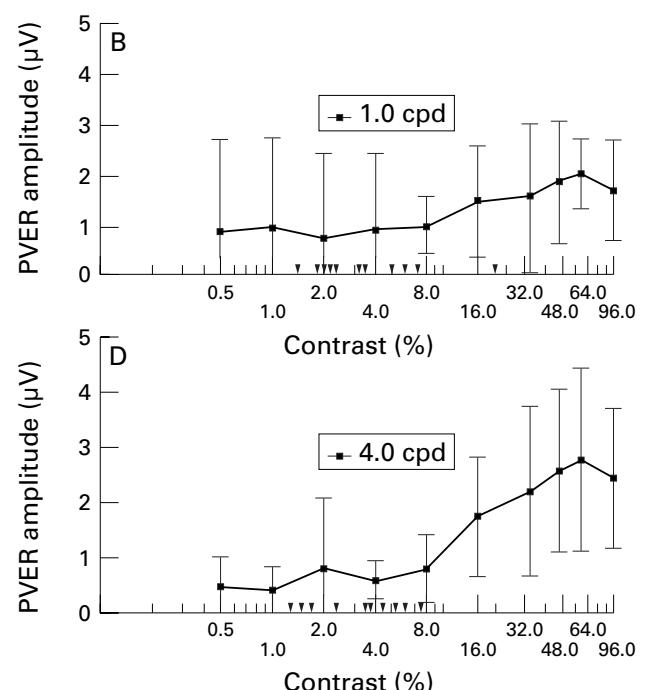

Figure 3 (A-E) Mean (SD) of the CSVER amplitudes plotted against 10 contrast levels in five different spatial frequencies $(0.5,1.0,2.0,4.0$, and 8.0 cycles per degree (cpd)). The arrows on the $x$ axis indicate the individual contrast thresholds (mean intercept of $0 \mu$ V on $x$ axis) obtained from the best fit regression.

nation of the contrast threshold at each spatial frequency generally took 30-60 seconds.

\section{MEASURING PSYCHOPHYSICAL CSF}

The gratings used were sinusoidally modulated and generated under computer control on a cathode ray display. The luminance $\left(50 \mathrm{~cd} / \mathrm{m}^{2}\right)$ was constant during the test. Using a $12 \times 12$ $\mathrm{cm}$ screen, a $6.8^{\circ}$ stimulus field size was obtained when viewed at a distance of $100 \mathrm{~cm}$ from the screen and a $5^{\circ}$ stimulus field size at a distance of $300 \mathrm{~cm}$. Sinusoidally modulated gratings of 31 contrast levels ranging from $98 \%$ to $0.2 \%$ were displayed in five spatial frequencies $(0.5,1.0,2.0,4.0,8.0 \mathrm{cpd})$ in gratings oriented at $90^{\circ}$ (vertical), $45^{\circ}$ (slanted to the right), and $135^{\circ}$ (slanted to the left). The levels were presented depending on the subject's response, by indicating the orientations using a three alternative, forced choice algorithm (that is, staircase procedure ${ }^{27}$ : each correct response decreased the contrast level $0.1 \mathrm{log}$ unit and each incorrect response increased the contrast level by 0.2 log unit), under a pseudorandom order. The threshold was computed as the contrast required for correct choices at a probability of $0.84 \%$.

STATISTICAL ANALYSIS

The intersubject homogeneity of the CSVER amplitude under different contrast conditions was tested via single factor repeated measures of analysis of variance (ANOVA). When the test was highly significant, Scheffe's test for multiple comparisons was applied. A p value $<0.05$ was considered to indicate statistical significance. 


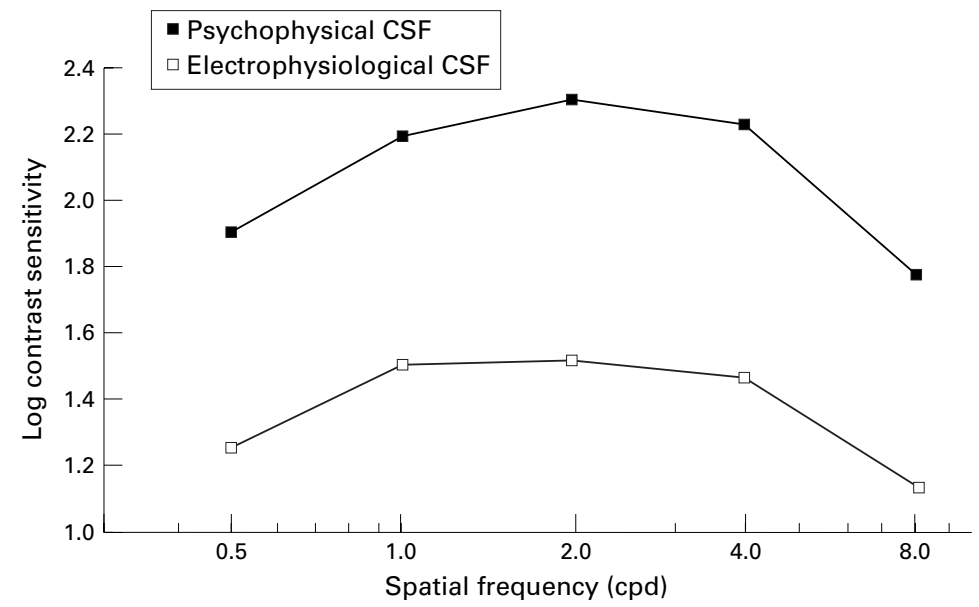

Figure 5 Comparison of the mean electrophysiological and psychophysical contrast sensitivity values at five different spatial frequencies. $c p d=$ cycles per degree.

\section{Results}

Figure 1(A-D) shows the CSVER amplitudes for the same normal subject at $0.5,2.0,4.0$, and $8.0 \mathrm{cpd}$ under 10 stimulus conditions. The mean PVER amplitude (95\% CI) was calculated with the DFT program. The contrast thresholds obtained were $0.96,1.67,1.59$, and $5.01 \%$ at $0.5,2.0,4.0$, and $8.0 \mathrm{cpd}$, respectively. The largest CSVER amplitude was recorded at $4.0 \mathrm{cpd}$, peaking at $48 \%$ of contrast (5.78 $\mu \mathrm{V}$ of the PVER amplitude).

Figure 2 shows the PVER amplitude $(\mu \mathrm{V})$ contrast stimulus level (\%) function at $0.5,1.0$, $2.0,4.0$, and $8.0 \mathrm{cpd}$. The amplitude represents the mean for 10 normal subjects. There was a general tendency for the CSVER amplitude to increase as the contrast increased. At $0.5 \mathrm{cpd}$, the CSVER amplitude increased continuously. However, at 1.0 to $8.0 \mathrm{cpd}$, the CSVER amplitude increased to the $64 \%$ contrast level, at which point the responses then decreased with a further increase in stimulus contrast. As a result, a high band pass filter curve was obtained.

The CSVER amplitudes (mean (SD)) of 10 different contrast stimuli $(0.5$ to $96 \%)$ at five spatial frequencies from 10 normal subjects are shown in Figure 3(A-E). At $0.5 \mathrm{cpd}$, the overall $F$ values from the repeated ANOVA measures to test the homogeneity of the

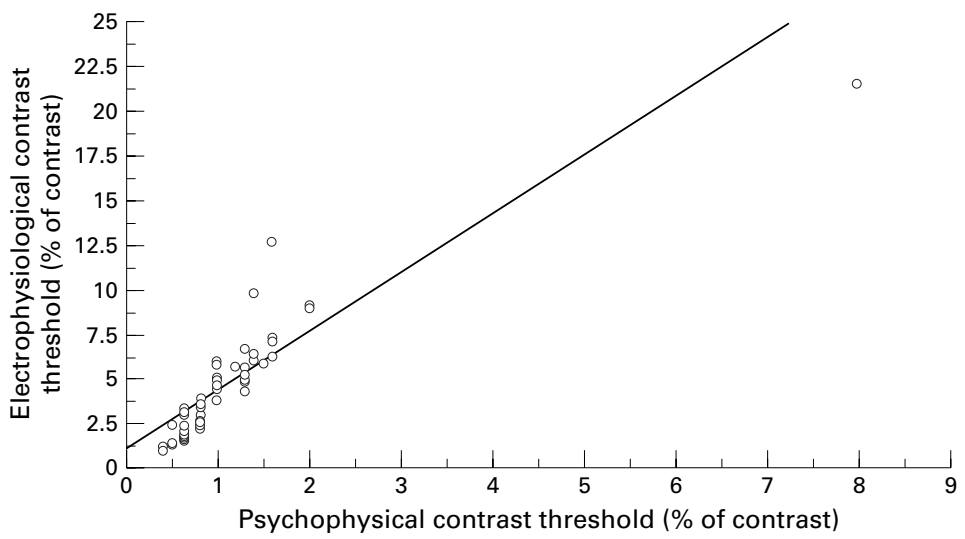

Figure 6 Scattergram showing a significant correlation between the individual psychophysical and electrophysiological contrast thresholds of 10 normal subjects at 0.5.1.0, 2.0, 4.0, and $8.0 \mathrm{cpd}$, under similar conditions. $r=0.89, p=0.0001$. responses were highly significant at contrasts up to $32 \%(F=4.08, p=0.0001)$. Statistically, at contrast levels higher than $32 \%$, the CSVER amplitude contrast stimuli function showed no difference. Similar findings were observed at $1.0,2.0,4.0$, and $8.0 \mathrm{cpd}$ with up to $32,32,32$, and $48 \%$ of contrast, respectively $(\mathrm{F}=5.47$, $\mathrm{p}=0.001 ; \quad \mathrm{F}=6.59, \quad \mathrm{p}=0.001 ; \quad \mathrm{F}=4.63$, $\mathrm{p}=0.0001 ; \mathrm{F}=3.86, \mathrm{p}=0.0002$, respectively). At these levels of contrast, we observed saturation phenomenon as a feature of CSVER.

Figure 4 shows the mean CSVER amplitudes of five spatial frequencies for the 10 contrast levels. A very distinct high pass filter function was observed at contrasts of 16 to $96 \%$, peaking at $4.0 \mathrm{cpd}$ with $64 \%$ contrast. At lower contrast levels $(0.5$ to $8 \%)$, this response pattern was not observed: at $8 \%$ and $4 \%$ of contrast, the peak was observed at $2.0 \mathrm{cpd}$; at $2 \%, 4.0 \mathrm{cpd}$; and at $1 \%$ and $0.5 \%$, a low pass filter curve was seen, peaking at $1.0 \mathrm{cpd}$. From 16 to $96 \%$, the PVER amplitudes were higher than in PVER derived from contrasts of $8 \%$ to $0.5 \%$ (approximately $0.5 \mu \mathrm{V}$ of amplitude voltage), in which the flattened shape is evident.

The mean electrophysiological (CSVER) values compared with the psychophysical sensitivities are shown in Figure 5. The curves have essentially the same band pass filter shape with the electrophysiological CSF being 0.65 , $0.68,0.79,0.76$, and $0.62 \mathrm{log}$ unit lower at 0.5, $1.0,2.0,4.0$, and $8.0 \mathrm{cpd}$, respectively. Both CSF peaked at $2.0 \mathrm{cpd}$ with the electrophysiological contrast threshold at $2.9 \%$ of contrast (CS, 34.5) and the psychophysical contrast threshold at $0.45 \%$ of contrast (CS, 222). The mean and intersubject variations (SD) were $1.26(0.175)$ at $0.5 \mathrm{cpd}, 1.522(0.247)$ at 1.0 $\mathrm{cpd}, 1.532(0.218)$ at $2.0 \mathrm{cpd}, 1.482(0.255)$ at $4.0 \mathrm{cpd}$, and $1.157(0.202)$ at $8.0 \mathrm{cpd}$ in the electrophysiological CSF, and $1.91(0.082)$ at $0.5 \mathrm{cpd}, 2.2(0.072)$ at $1.0 \mathrm{cpd}, 2.321(0.107)$ at $2.0 \mathrm{cpd}, 2.242(0.202)$ at $4.0 \mathrm{cpd}$, and 1.78 $(0.286)$ at $8.0 \mathrm{cpd}$ in the psychophysical method.

The statistical correlation between the individual values of both contrast thresholds (psychophysical and electrophysiological) is showed as a scattergram (Fig 6) at 0.5, 1.0, 2.0, 4.0 , and $8.0 \mathrm{cpd}$ for 10 normal subjects. The solid line represents the linear regression between the two contrast thresholds. The correlation coefficient for CSVER $v$ psychophysics was approximately 0.89 indicating that the contrast threshold derived from the VER provided good prediction of the conventional (psychophysical) threshold under similar conditions $(p=0.0001)$.

\section{Discussion}

The importance of the contrast sensitivity (CS) evaluation lies in its ability to detect visual function abnormalities in patients who, despite a good Snellen acuity, complain of visual disturbances. These patients often complain of "misty" vision. Isolated CS losses are present in certain diseases, such as optic nerve disease, and often the loss is more prominent and disturbing to the patient than is a decrease in 
visual acuity. The clinical applications of CSF are discussed in review articles by Sekuler, ${ }^{28}$ and DeValois and DeValois. ${ }^{29}$

Campbell and Robson ${ }^{1}$ interpreted their psychophysical CSF results as indicating the existence of "multiple or separate channels" within the visual system, which are selectively sensitive to a narrow spatial frequency. The resultant CSF was an inverted U-shaped curve, peaking at approximately $5.0 \mathrm{cpd}$. The concept of separate channels for different spatial frequencies is in a sense a restatement of the fact that the retina is not uniform. Channels may be the expression of single classes of neuronal function. The activities of ganglion cell subpopulations in the visual pathway can be isolated by different spatial frequencies. There has been much speculation concerning the source of the central/peripheral field origin ${ }^{11}$ to cortical manifestation of the magnocellular and parvocellular pathways, ${ }^{21}{ }^{30-32}$ including the suggestion that they represent separate motion and pattern discrimination mechanisms. ${ }^{33}$ Only the fovea is specialised for high visual acuity and must, therefore, process all information involving high spatial frequencies. In the retinal periphery, only lower frequency channels are represented. ${ }^{7}$ The clinical value of this test is that it allows the rapid assessment of peripheral (low spatial frequencies) and central retinal function (high spatial frequencies).

In the present study, we estimated the CSF values derived from steady state visual evoked potentials (VEPs) and also psychophysically under similar conditions. In electrophysiological recordings, we observed saturation phenomena as a feature of the contrast VER. This phenomenon may be influenced by several conditions such as temporal and spatial frequencies, the mean retinal luminance, the type of pattern, and the size of the pattern elements. ${ }^{34}$ Contrast VER recordings showed the saturation phenomena at $32 \%$ or $48 \%$ of contrast at the different spatial frequencies tested, in agreement with previous studies. ${ }^{12}{ }^{34}$ The CSVER recorded in the present investigation showed a nearly linear function, which allowed the extrapolation to zero voltage and the rapid measurement of the contrast threshold, thus confirming the results of other studies. ${ }^{112035}$ By plotting both psychophysical and electrophysiological CSF against five different spatial frequencies under similar conditions, we observed nearby parallel inverted U-shaped functions and electrophysiological CSF lower by 0.62 to $0.79 \mathrm{log}$ unit at all spatial frequencies. Previous studies demonstrated a good correlation between CSF by steady state VEP and by the psychophysical method. ${ }^{18} 2030$ 36-41 The electrophysiological CSF was consistently less sensitive than the psychophysical approach. The methodology of our study does not permit us to clarify mechanisms of responses involved in both visual pathways. As is well known, the psychophysical threshold is a perception in which only a few neurons need to be activated to obtain the threshold, whereas a greater number of them need to be activated to produce evident electrical potentials in the occipital cortex during the electrophysiological test. Additionally, stimulus field is a very important factor: the size of the stimulus field and the number of elements (number of check sizes when the checkerboard pattern is used and number of gratings when gratings are used as visual stimuli). Thus, it seems that there is certain correlation between the number of elements and the magnitude of the responses, especially in the case of PVER. ${ }^{42}$ Ideally, it would have been best to use similar stimulus field sizes in both the psychophysical and electrophysiological tests. However, the limitation of PVER meant that a relatively large stimulus field size had to be used in order to record good response at low spatial frequencies. Differences between the experiments, especially in the stimulus field size, grating orientation, and temporal modulation, are factors that cannot be ignored. Allen et $a l^{20}$ tested different electrode positions for the measurement of contrast thresholds derived from sweep VER and compared the results with those obtained by the psychophysical method. Using a similar technique (frame rate of $7.5 \mathrm{~Hz}$, contrast levels that increased from 0.5 to $40 \%$ during the trial every 0.5 second and a mean luminance of $80 \mathrm{~cd} / \mathrm{m}^{2}$ ), the PVER amplitudes obtained were less (up to $1.0 \mu \mathrm{V}$ ) than that recorded in this study. In addition, in the present investigation, the CSF derived from the electrophysiological method had slightly higher intersubject variation than did the values obtained by psychophysical method. The results confirm those of Cannon, ${ }^{18}$ who used checkerboard patterns, and Allen et al ${ }^{20}$ who showed that the standard deviation for the sweep VEP was not significantly greater than the corresponding values by psychophysical approach.

In summary, we have described an objective technique for measuring CSF, which correlates well with the psychophysical method. The intersubject difference was reliable, the evoked responses were reproducible, and the test was rapidly performed (20 seconds at each spatial frequency). Further studies are needed to evaluate the use of this technique in uncooperative patients. In view of the relation between contrast sensitivity and visual performance, the ease with which CSF may be determined from the VEP is likely to prove to be a valuable tool in the clinical setting, particularly in paediatric vision evaluation, and in visual research.

We thank Charles L Schepens, MD, for his support of this project and Neil Winchester and Harold Goldstein of Neuroscientific Corp (Farmingdale, NY) for their help in developing the SPVER program. We are also grateful to Lynda C Charters of Medical International (Marlboro, MA) and Dr Stephen Hyslop for editing the manuscript and to Steven A Burns, $\mathrm{PhD}$, for his critical review of the work.

\footnotetext{
1 Campbell FW, Robson JG. Application of Fourier analysis to the visibility of gratings. F Physiol 1968;203:551-6.

2 Enroth-Cugell C, Robson JG. The contrast sensitivity of retinal ganglion cells of the cat. F Physiol 1966;187:517-52. 3 Bodis-Wollner I. Visual acuity and contrast sensitivity in patients with cerebral lesions. Science 1972;178:769-71.

4 Regan D, Silver R, Murray TJ. Visual acuity and contrast sensitivity in multiple sclerosis-hidden visual loss: an auxiliary diagnostic test. Brain 1977;100:563-79.

5 Hess RF, Howell ER. The threshold contrast sensitivity function in strabismic amblyopia: evidence for a two type classification. Vision Res 1977;17:1049-55.

6 Sokol S, Moskowitz A, Skarf B, et al. Contrast sensitivity in diabetics with and without background retinopathy. Arch Ophthalmol 1985;103:51-4.
} 
7 Arden GB. The importance of measuring contrast sensitivity in cases of visual disturbance. $\mathrm{Br} \mathcal{F}$ Ophthalmol 1978;62: ity in cases

8 Bodis-Wollner I, Hendley CD, Mylin LH, Thornton J. Evoked potentials and the visuogram in multiple sclerosis. Ann Neurol 1979;5:40-7.

9 Regan D, Neima D. Low contrast letter charts as a test of visual function. Ophthalmology 1983;90:1192-200.

10 Ginsburg AP. A new contrast sensitivity vision test chart. Am $\mathcal{F}$ Optom Physiol Opt 1984;61:403-7.

11 Campbell FW, Maffei L. Electrophysiological evidence for the existence of orientation and size detectors in the human visual system. F Physiol 1970;207:635-52.

12 Spekreijse H. Analysis of EEG responses to diffuse to patterned light in the human. Thesis, University of Amsterdam. The Hague: DW Junk, 1966.

13 Daniel PM, Whitteridge D. The representation of the visual field on the cerebral cortex in monkeys. F Physiol 1961;159: 203-21.

14 Rovamo J, Virsu V. An estimation and application of the human cortical magnification factor. Exp Brain Res human cortical

15 Katsumi O, Van de Velde FJ, Mehta MC, Hirose T. Topographical analysis of peripheral vs central retina with pattern reversal visual evoked response and the scanning laser ophthalmoscope. Acta Ophthalmol 1991;69:596-602.

16 Katsumi O, Tetsuka H, Tetsuka S, Hoon-Ohn Y, Hirose T. Effects of light scatter, defocusing, mean luminosity, contrast and central scotoma on the PVER amplitudecheck size function curve. Acta Ophthalmol 1993;71:60615.

17 Nakayama K, Mackeben M. Steady state visual evoked potentials in the alert primate. Vision Res 1982;22:126171 .

18 Cannon MW. Contrast sensitivity: psychophysical and evoked potentials methods compared. Vision Res $1983 ; 23$ 87-95.

19 Katsumi O, Tanino T, Hirose T. Measurement of contrast sensitivity function using pattern-reversal visual evoked
responses. Graefes Arch Clin Exp Ophthalmol 1985;223: respons $190-5$.

20 Allen D, Norcia AM, Tyler CW. Comparative study of electrophysiological and psychophysical measurement of the contrast sensitivity function in humans. Am F Optom Physio Opt 1986;63:442-9.

21 Tyler CW, Apkarian PA. Effects of contrast, orientation and binocularity on the pattern evoked potential. Vision Res 1985;25:755-66.

22 Howe JW, Mitchell KW. The objective assessment of contrast sensitivity function by electrophysiological means. Br f Ophthalmol 1984;68:626-38.

23 Regan D. Speed assessment of visual acuity in amblyopia by the evoked potential method. Ophthalmologica 1977;175: 156-64.
24 Tyler CW, Apkarian PA, Levi DM, Nakayama K. Rapid assessment of visual function:an electronic sweep technique for the pattern evoked potential. Invest Ophthalmol -13.

25 Norcia AM, Tyler CW. Spatial frequency sweep VEP:visual acuity during the first year of life. Vision Res 1985;25:1399408.

26 Norcia AM, Tyler CW, Hamer RD. High visual contrast sensitivity in the young human infant. Invest Ophthalmol Vis Sci 1988;29:44-9.

27 Wetherhill GB, Levitt H. Sequential estimation of points on a psychometric function. Br F Math Stat Psychol 1960;18:110 .

28 Sekuler R. Spatial vision. Anпu Rev Psychol 1974;25:21532.

29 DeValois RL, DeValois KK. Spatial vision. Anun Rev Psychol 1980;31:309-41.

30 Bobak P, Bodis-Wollner I, Harnois C, Thornton J. VEPs in humans reveal high and low spatial contrast mechanisms. Invest Ophthalmol Vis Sci 1984;25:980-3.

31 Hubel DH, Livingstone MS. Color and contrast sensitivity in the lateral geniculate body and primary visual cortex of the macaque monkey. F Neurosci 1990;10:2223-37.

32 Merigan WH, Katz LM, Maunell JHR. The effects of parvocellular lateral geniculate lesions on the acuity and contrast sensitivity of macaque monkeys. I Neurosci 1991;11:994-1001.

33 Murray IJ, Kulikowski JJ. VEPs and contrast. Vision Res 1983;23:1741-3.

34 Spekreijse H, Twell Lh van der, Zuidema T. Contrast evoked responses in man. Vis Res 1973;13:1577-601.

35 Campbell FW, Kulikowski JJ. The visual evoked potential as a function of contrast of a grating pattern. F Physiol $1972 ; 222: 345-56$

36 Harris L, Atkinson J, Braddick O. Visual contrast sensitivity of a 6-month-old infant measured by the evoked potential. Nature 1976;264:570-1.

37 Bodis-Wollner I. Vulnerability of spatial frequency channels in cerebral lesions. Nature 1976;261:309-11.

38 Bodis-Wollner I. Recovery from cerebral blindness: evoked potential and psychophysical measurements. Electroenceph potential and psychophysical mea
Clin Neurophysiol 1977;42:178-84.

39 Pirchio M, Spinelli D, Fiorentini A, Maffei L. Infant contrast sensitivity evaluated by evoked potentials. Brain 1978;141:179-84

40 Fiorentini A, Pirchio M, Spinelli D. Scotopic contrast sensitivity in infants evaluated by evoked potentials. Invest $O p h$ thalmol Vis Sci 1980;19:950-5.

41 Seiple HS, Kupersmith MJ, Nelson JI, Carr RE. The assessment of evoked potential contrast threshold using real-time retrieval. Invest Ophthalmol Vis Sci 1984;25:627-31.

42 Katsumi O, Hirose T, Tanino T. Effect of stimulus field size and the localization on the binocular pattern reversal visual evoked response. Doc Ophthalmol 1988;69:293-305. 